Randomised controlled trial

The therapeutic value of hypertonic saline in acute bronchiolitis remains unclear

\subsection{6/ebmed-2014-110082}

\section{Jay Pershad}

Division of Emergency Medicine, Department of Pediatrics, University of Tennessee Health Science Center and Le Bonheur Children's Hospital, Memphis, Tennessee, USA

Correspondence to: Professor Jay Pershad, Division of Pediatric Emergency Medicine, University of Tennessee Health Science Center and Le Bonheur Children's Hospital, 50 N Dunlap, St. Memphis, TN 38103, USA; jay.pershad@mlh.org

Commentary on: Wu S, Baker C, Lang ME, et al. Nebulized hypertonic saline for bronchiolitis: a randomized clinical trial. JAMA Pediatr 2014;168:657-63 and Florin TA, Shaw KN, Kittick M, et al. Nebulized hypertonic saline for bronchiolitis in the emergency department: a randomized clinical trial. JAMA Pediatr 2014;168:664-70.

\section{Context}

Bronchiolitis is common in children below 2 years of age and is a leading cause of infant hospitalisation, accounting for $\$ 1.73$ billion in hospital charges in the USA annually. ${ }^{1}$ The putative effect of hypertonic saline (HTS) in bronchiolitis is to absorb mucosal water, hydrate the airwaysurface liquid in the bronchioles and enhance mucociliary clearance. ${ }^{2}$ However, the therapeutic value of HTS in acute bronchiolitis remains unclear.

\section{Methods}

Both randomised controlled trials (RCTs) were conducted in urban, tertiary level, paediatric emergency departments (ED) in the USA. They excluded children with significant comorbidity, and compared the effect of nebulised 3\% HTS with normal saline (NS) controls. Both studies used albuterol, given either just prior to or within $90 \mathrm{~min}$ of HTS.

\section{Findings}

Florin and colleagues enrolled 31 patients in each group, concluding that improvement in Respiratory Assessment Change Score (RACS; primary outcome) $1 \mathrm{~h}$ after intervention was less in the HTS group than the NS group (difference in mean $\mathrm{RACS}=2.5,95 \%$ CI 0.5 to 4.6). A change in RACS of three points was deemed clinically significant. Wu and colleagues enrolled 231 and 216 patients into the HTS and NS groups, respectively. They concluded that the HTS group had a lower admission rate (28.9\% vs $42.6 \%$; OR=0.45, 95\% CI 0.28 to 0.86 ), but did not observe a statistically significant difference in length of stay (LOS) or RACS. In both studies, additional therapies were administered at physician discretion and no significant difference in secondary outcomes (eg, adjunctive therapy with oxygen, use of steroids or adverse events) was noted. Mean duration of illness in each study was 3.4 and 5 days, respectively.

\section{Commentary}

These RCTs were well designed, with low overall risk of bias, albeit with some imperfections. Wu and colleagues' study was underpowered to detect a planned difference of 30\% in admission rate or $24 \mathrm{~h}$ in LOS. There was no objective severity of illness criteria for inclusion, admission or discharge readiness, making clinician bias a potential issue. Florin and colleagues included a wide severity-of-illness range (Respiratory Distress Assessment Index (RDAI)4-15) and utilised the RACS (calculated using change in RDAI and respiratory rate), as short-term proxy outcome for need for hospitalisation. Their study was not powered for detection of a change in admission rate.

The RDAI has poor discriminative and construct validity in predicting hospitalisation and LOS in bronchiolitis, in part because it does not include respiratory rate or $\mathrm{O}_{2}$ saturation, both important variables for a clinician to determine disposition. ${ }^{3} \mathrm{Wu}$ and colleagues reaffirm this limitation-although they reported no significant difference in mean pretreatment and post-treatment RDAI scores in the two groups, the difference in admission rate was statistically significant.

The most recent meta-analysis on HTS in bronchiolitis seems promising in terms of LOS reduction for mild-to-moderate disease (mean=1.15 days, 95\% CI 1.49 to 0.82). However, the benefit appears to be concentrated in European studies in which mean LOS was relatively long (5-7 days), rendering data less generalisable to US populations where mean LOS is 2-3 days. ${ }^{4}$ This same meta-analysis included four ED-based trials that did not show any significant short-term (30-120 min) improvement in clinical score and oxygen saturation with up to three doses of nebulised 3\% saline. Trials published since this meta-analysis have also not demonstrated benefits of using HTS. What we can glean from the literature is that no significant adverse events were noted with use of 3\% or higher concentrations of HTS. ${ }^{5-9}$

Bronchodilators have been used in conjunction with HTS in several trials in an effort to mitigate bronchospasm purportedly related to HTS, even though by themselves they have not been shown to improve outcomes. ${ }^{1}{ }^{4}$ Indeed, we cannot rule out a possible contribution of HTS effect on increasing albuterol action. It has also been suggested that those with an individual or close family history of atopy may preferentially benefit from $\beta$-agonists, with NS or HTS. In an RCT conducted on infants with moderate bronchiolitis in the ED setting, although the combination of salbutamol with HTS did not lead to an improved bronchiolitis severity score, atopic children preferentially benefited from combined salbutamol/NS. ${ }^{9}$

Where does this leave acute care clinicians? There are three questions we need to ask. First, what are the confounding issues in interpreting RCTs of HTS in acute bronchiolitis? There is much heterogeneity with regard to study setting (ED vs inpatient), severity of illness, HTS concentration, adjunctive bronchodilator use, severity of illness scoring systems, frequency of drug administration, co-interventions such as suction or supplemental $\mathrm{O}_{2}$, and criteria for admission or discharge readiness. Second, what is a measurable, objective and relevant clinical outcome? In a disease expected to last several days, a short-term improvement in clinical score is less important than an impact on admission rate and LOS. Finally, how do clinicians manage acute bronchiolitis when the season changes and we get inundated with patients?

My recommendation-part evidence-based and part experiential-for infants without significant comorbidity is as follows: for mild disease, masterly inactivity with close observation at home is prudent. For patients with severe respiratory distress, dehydration or hypoxaemia, hospitalise and provide necessary supportive care. Infants with moderate disease are the focus of controversy. In general, if symptom duration is $72 \mathrm{~h}$ or more, and the infant is feeding well and maintaining adequate oxygenation, doing less may be most effective. Other than nasal suction for the obligatory nose breathers, the additional cost and resources of nebulisation may not be justified. If symptom duration is less than $72 \mathrm{~h}$, with predominant wheeze (rather than rales) and features of atopy, a trial of $\beta$-agonist with $3 \%$ or 5\% HTS may be justified. If there is improvement after 30-60 min, 
one could continue this therapy and then decide final disposition after a period of observation and feeding.

We are in a state of clinical equipoise regarding the role of HTS in moderate bronchiolitis. Placebo-controlled (except 0.9\% saline), multicentre trials conducted with clinically meaningful primary outcomes and a longer follow-up period are now required to determine the efficacy of HTS in bronchiolitis.

\section{Competing interests None.}

Provenance and peer review Commissioned; internally peer reviewed.

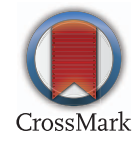

\section{References}

1. Hasegawa K, Tsugawa Y, Brown DF, et al. Trends in bronchiolitis hospitalizations in the United States, 2000-2009. Pediatrics 2013;132:28-36.
2. Mandelberg A, Amirav I. Hypertonic saline or high volume normal saline for viral bronchiolitis: mechanisms and rationale. Pediatr Pulmonol 2010;45:36-40.

3. Destino L, Weisgerber MC, Soung $P$, et al. Validity of respiratory scores in bronchiolitis. Hosp Pediatr 2012;2:202-9.

4. Zhang L, Mendoza-Sassi RA, Wainwright C, et al. Nebulised hypertonic saline solution for acute bronchiolitis in infants. Cochrane Database Syst Rev 2013;7: CD006458.

5. Jacobs JD, Foster M, Wan J, et al. 7\% Hypertonic saline in acute bronchiolitis: a randomized controlled trial. Pediatrics 2014;133:e8-13.

6. Nenna R, Papoff P, Moretti C, et al. Seven percent hypertonic saline-0.1\% hyaluronic acid in infants with mild-to-moderate bronchiolitis. Pediatr Pulmonol 2014;49:919-25.

7. Teunissen J, Hochs AH, Vaessen-Verberne A, et al. The effect of 3\% and 6\% hypertonic saline in viral bronchiolitis: a randomised controlled trial. Eur Respir J Published Online First: 25 Jun 2014. doi:10.1183/09031936.00159613

8. Sharma BS, Gupta MK, Rafik SP. Hypertonic (3\%) saline vs 0.93\% saline nebulization for acute viral bronchiolitis: a randomized controlled trial. Indian Pediatr 2013;50:743-7.

9. Ater D, Shai H, Bar BE, et al. Hypertonic saline and acute wheezing in preschool children. Pediatrics 2012;129:e1397-403. 